\title{
Computing Generalized Linear Model using Iteratively Weighted Least Squares and Coordinate Descent
}

\author{
Khoa Huynh ${ }^{1}$ and Xia Wang ${ }^{1}$ \\ ${ }^{1}$ Department of Mathematical Sciences Division of Statistics and Data Science, University \\ of Cincinnati
}

January 21, 2021

\section{Hosted file}

manuscript.pdf available at https://authorea.com/users/390992/articles/505196-computinggeneralized-linear-model-using-iteratively-weighted-least-squares-and-coordinate-descent 